\title{
Equation of state for QCD matter in a quasiparticle model
}

\author{
R. Schulze† B. Kämpfer \\ Forschungszentrum Dresden-Rossendorf, PF 510119, 01314 Dresden, Germany \\ Institut für Theoretische Physik, TU Dresden, 01062 Dresden, Germany
}

\begin{abstract}
A phenomenological QCD quasiparticle model provides a means to map lattice QCD results to regions relevant for a variety of heavy-ion collision experiments at larger baryon density. We report on effects of collectives modes and damping on the equation of state.
\end{abstract}

Strongly interacting matter is governed by the fundamental theory of QCD, which can be solved numerically using Monte-Carlo calculations on the lattice. However, reliable results are still limited to rather small net baryon densities [1]. As an alternative approach to obtain thermodynamic gross properties of the quark-gluon plasma, a thermodynamic quasiparticle model (QPM) incorporating 1-loop QCD in hard thermal loop (HTL) approximation can be utilized [2, 3, 4, 5].

Employing [5] the Cornwall-Jackiw-Tomboulis formalism, the entropy density assumes the simple form of a sum $s=s_{g, \mathrm{~T}}+s_{g, \mathrm{~L}}+s_{q, \mathrm{Pt} .}+s_{q, \mathrm{Pl}}+s^{\prime}$ over partial entropy density contributions from four quasiparticle excitations (transverse and longitudinal gluons, quarks and plasminos). The residual interaction term $s^{\prime}$ vanishes at 2-loop order for the generating functional. Individual contributions read

$$
s_{i} \sim d_{i} \int_{\mathrm{d}^{4} k()}\left\{\pi \varepsilon\left(\operatorname{Im} D_{i}^{-1}\right) \Theta\left(\xi_{i} \operatorname{Re} D_{i}^{-1}\right)-\arctan \frac{\operatorname{Im} \Pi_{i}}{\operatorname{Re} D_{i}^{-1}}+\operatorname{Re} D_{i} \operatorname{Im} \Pi_{i}\right\},
$$

where $\int_{\mathrm{d}^{4} k()}$ represents the convolution of the parentheses \{\} with the derivatives of the distribution functions with respect to the temperature $T$, i.e. $\int \mathrm{d}^{3} k \int_{-\infty}^{\infty} \mathrm{d} \omega /(2 \pi)^{4}\left(\partial n_{\mathrm{B}} / \partial T\right)$ for the gluons and $\int \mathrm{d}^{3} k \int_{0}^{\infty} \mathrm{d} \omega /(2 \pi)^{4}\left(\partial n_{\mathrm{F}} / \partial T+\partial n_{\mathrm{F}}^{\mathrm{A}} / \partial T\right)$ for quarks and plasminos (superscript A for antiparticles). The sign constant $\xi_{i}$ is -1 for quasiparticles with real particle interpretation (transverse gluons and quarks) and +1 for the collective modes (longitudinal gluons and plasminos). $D_{i}\left(\Pi_{i}\right)$ stands for the propagators (self-energies) of species $i$. From the entropy density, the remaining state variables can be constructed in a self-consistent manner.

To describe results of lattice QCD calculations at zero chemical potential, a temperature shift is introduced into the running coupling $g^{2}$ changing it to an effective coupling $G^{2}$; the parameters of the coupling (a scale parameter and the temperature shift) are then adjusted to the lattice data.

To obtain the coupling $G^{2}$ at nonzero chemical potential $\mu$, the self-consistency of the model and the stationarity of the thermodynamic potential are employed, leading to a quasilinear partial differential equation for the coupling (dubbed flow equation)

$$
a_{T} \frac{\partial G^{2}}{\partial T}+a_{\mu} \frac{\partial G^{2}}{\partial \mu}=b
$$



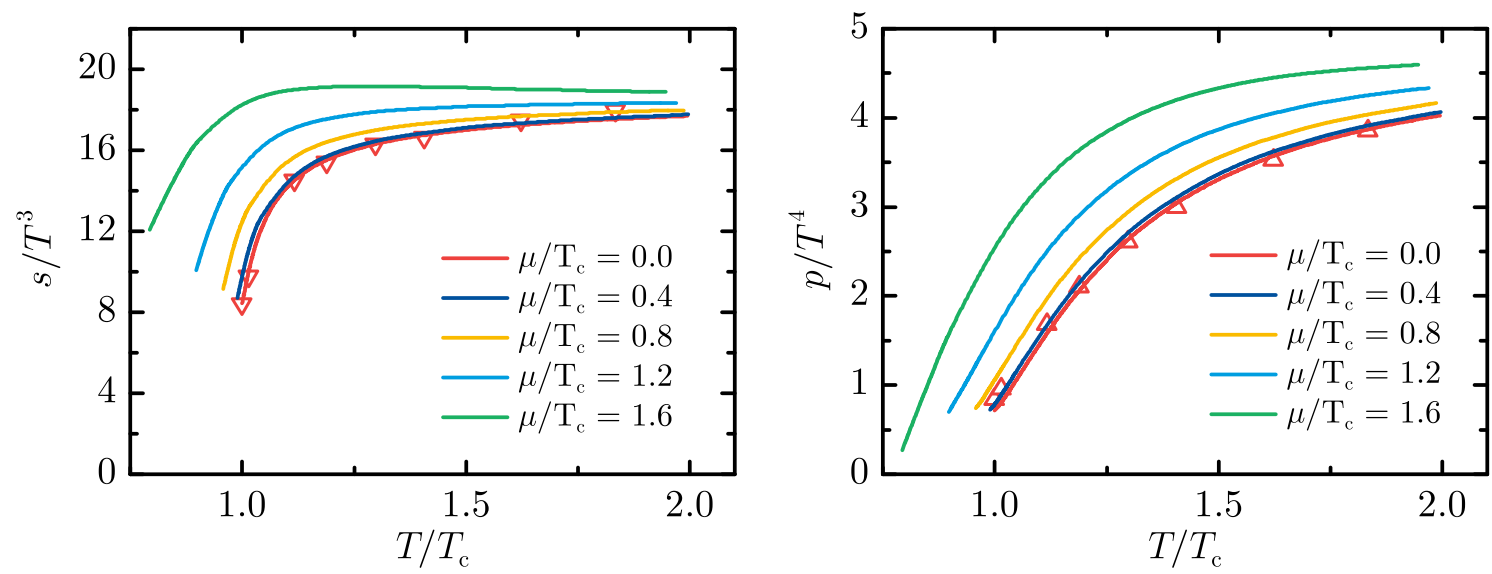

Figure 1: Scaled entropy density $s / T^{3}$ (left) and pressure $p / T^{4}$ (right) of $2+1$ quark flavors as functions of the scaled temperature $T / T_{c}$ for several values of the quark chemical potential $\mu$. Lattice data (symbols) for $\mu=0$ from [6]. The termination of the curves at $T \leq T_{c}$ is at the conjectured transition line to a confined state, cf. [5, 7].

with coefficients $a_{T, \mu}$ and $b$ listed in [5]. This is the HTL QPM, as the HTL approximation is used for dispersion relations.

For simplified versions of the HTL QPM, e.g. neglecting collective modes, the solution of the flow equation leads to ambiguities. It was shown that collective modes and Landau damping as well as the use of the momentum-dependent HTL dispersion relations are essential to preserve the self-consistency of the model [7]. Utilizing the full model, thermodynamic gross properties of the quark-gluon plasma can be obtained. As an example, the entropy density and pressure along lines of constant chemical potential are exhibited in Figure 1, From these state quantities, it is possible to provide an equation of state for present and upcoming heavy-ion experiments such as at RHIC, LHC [8], SPS and FAIR. In particular at FAIR the baryon density effects covered by our model become severe.

One author (RS) thanks the organizers of the conference for support and the opportunity to present his results.

\section{References}

[1] S. Ejiri, F. Karsch, E. Laermann, and C. Schmidt, Phys. Rev. D 73 (2006) 054506

[2] A. Peshier, B. Kämpfer, and G. Soff, Phys. Rev. C 61 (2000) 045203

[3] J.-P. Blaizot, E. Iancu, and A. Rebhan, Phys. Rev. D 63 (2001) 065003

[4] M. Bluhm, B. Kämpfer, R. Schulze, and D. Seipt, Eur. Phys. J. C 49 (2007) 205

[5] R. Schulze, M. Bluhm, and B. Kämpfer, Eur. Phys. J. ST 155 (2008) 177

[6] F. Karsch, J. Phys. G 34 (2007) S627

[7] R. Schulze, Quasiparticle description of QCD thermodynamics: effects of finite widths, Landau damping and collective excitations, Diploma thesis, Technical University Dresden (2007)

[8] M. Bluhm, B. Kämpfer, R. Schulze, D. Seipt, and U. Heinz, Phys. Rev. C 76 (2007) 034901

\footnotetext{
${ }^{\dagger}$ r.schulze@fzd.de
} 